\title{
EIN NEUER FARAZEITLICHER FELDKAUFVERTRAG IN JAPAN
}

\section{Tohru Gomi*}

Seit den zwei zusammenfassenden Behandlungen der aus Fara stammenden Rechtsurkunden der Fara-zeit von Edzard und Krecher(1) hat die Zahl der Urkunden, die uns für das Studium der damaligen Gesellschaft zur Verfügung stehen, allmählich gestiegen, sie bleibt doch nach wie vor recht gering trotz einiger neuen additionellen Veröffentlichungen der Urkunden von Farber und Grégoire in diesen Jahren.(2) Jeder neue Text kann also, wie Herr und Frau Farber in WO 8, S. 178 sagen, schon an sich wert sein, veröffentlicht zu werden.

Der vorliegende Text fand sich einmal im Besitz des Institute of Oriental Culture (Tōyō-Bunka-Kenkyūjo) der Universität Tokyo; er bleibt bis heute unveröffentlicht. Nach der Erklärung des Herrn T. Matsutani (außerordentlicher Professor) der Universität, und der beiden Herren Y. Chiyonobu und M. Furuyama (Forscher am Institut), spielten dabei verschiedene komplizierte Umstände eine Rolle. Als 1956 die Universität eine Expeditionsmannschaft nach dem Iran und dem Irak absandte, becam sie von einem damals in der Stadt Bagdad wohnhaften Japaner, einige Dinge, darunter war eine Tontafel, die seitdem in den Besitz des Instituts kam. Aber danach gab das Institut unter einigen Umständen das Besitzrecht der wertvollen Tontafel auf. Das Original der Tafel ist also derzeit nicht im Institut.

Aber es war glücklich, daß bei einer Ausstellung des Erworbenen durch die Expedition im Jahre 1967, das Institut eine ziemlich große Anzahl Abdrucke der Tafel machen ließ. Drei davon kamen in die Hände von Herren Matsutani, Chiyonobu und Furuyama und sind bei ihnen bis heute intakt aufbewahrt. Diese drei Abdrucke sind so vollständig und, nur von einigen Zeilen abgesehen, fast ganz lesbar, daß es uns möglich scheint, ohne Hilfe der originalen Tafel deren Text grundsetzlich $\mathrm{zu}$ verstehen.

Der Text wird hier mit der freundlichen Erlaubnis der oben erwähnten Eigentümer der Abdrucke, die eine schnelle Veröffentlichung wünschen,

* Außorordentlicher Professor der literarischen Fakultät, Shizuoka-Mädchenhochschule. 
publiziert. Dafür möchte ich ihnen herzlich danken. Mein besonderer Dank gilt auch Prof. Sh. Fukai an der Tokyo-Universität für seine Freundlichkeit, mir die im Institut aufbewahrten Photos des Originals zur Verfügung zu stellen, die meine Arbeit viel erleichterten.

Die Größe der Abdrucke ist $81 \mathrm{~mm}$ (Länge) $\times 81 \mathrm{~mm}$ (Breite) $\times 16 \mathrm{~mm}$ (Dicke). Die Zeilen II 4-5 der Vorderseite sind höchst angeschwollen; dagegen ist die dreikolumnige Rückseite fast eben nur mit einigen Abdrücken der Finger des Schreibers. Dafür, daß die originale Tafel nicht nachgemacht ist, sprechen viele innere und äußere Kriterien wie die Größe und Form der Tafel, die Form und Schreibung der Zeichen usw.

Unten sind meine autographierte Kopie und eine provisorische Umschrift und Übersetzung des Textes.

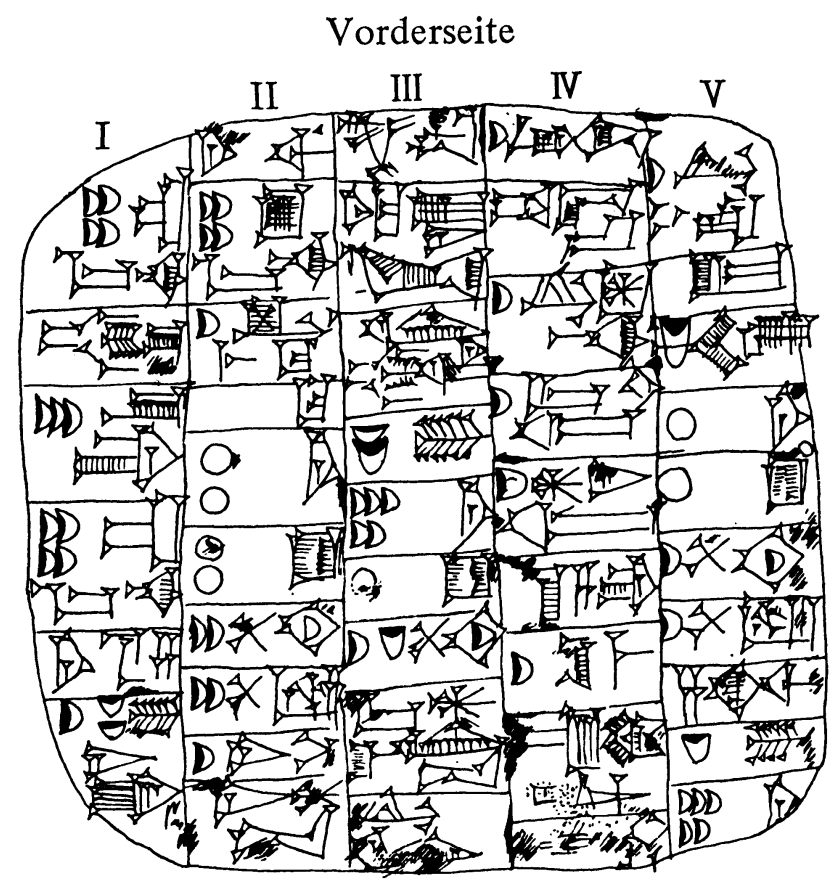




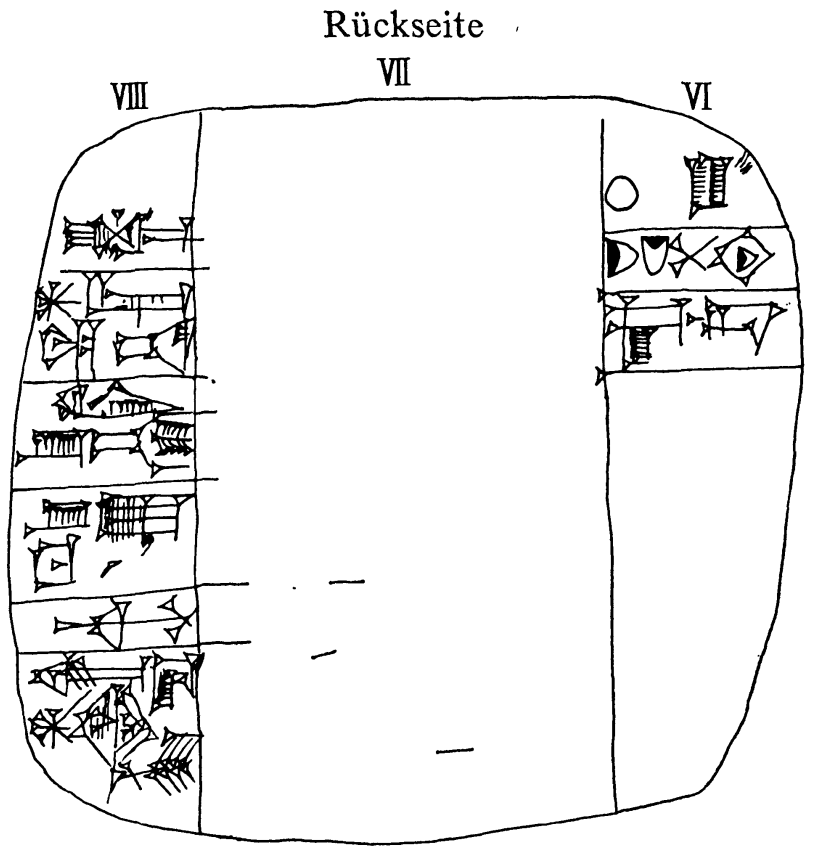

(Vs.)

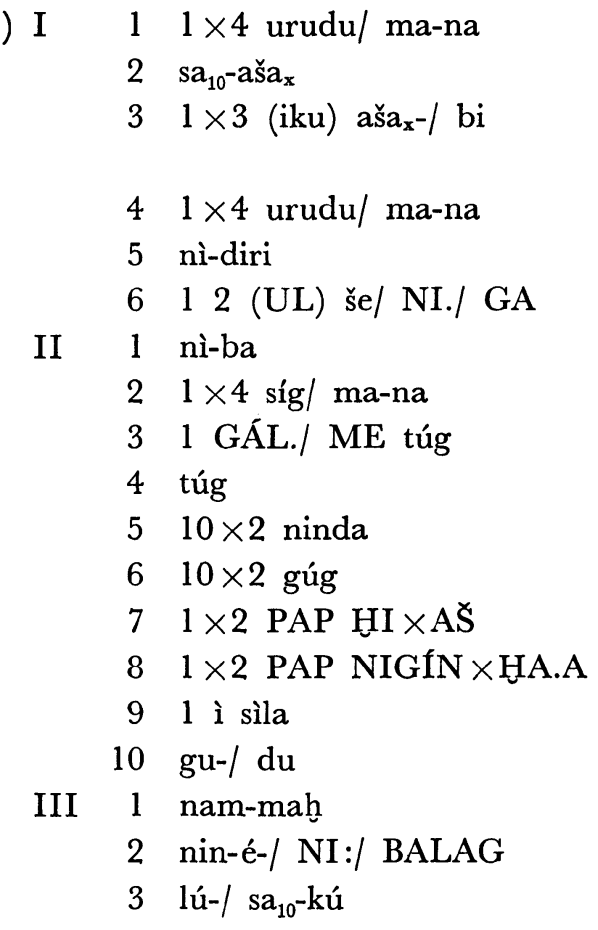

4 Mana Kupfer (sind)

Kaufpreis für das Feld.

3 Iku (sind der Flächenraum) des Feldes.

4 Mana Kupfer (sind) zusätzliche (Erstattungs-) Zahlung.

1 NI. GA und 2 UL Gerste (sind)

Geschenk.

4 Mana Wolle,

1 Stück ... -Gewand;

(die beiden sind) Stoffe?;

20 Brote

20 "Gebäcke,"

2 PAP H.,

2 PAP N.,

(und) 1 Sila Fett/Öl (sind)

(für) Gudu,

Nammah

(und) Nin'ebalagni,

die Leute, die den Kaufpreis "ge- 


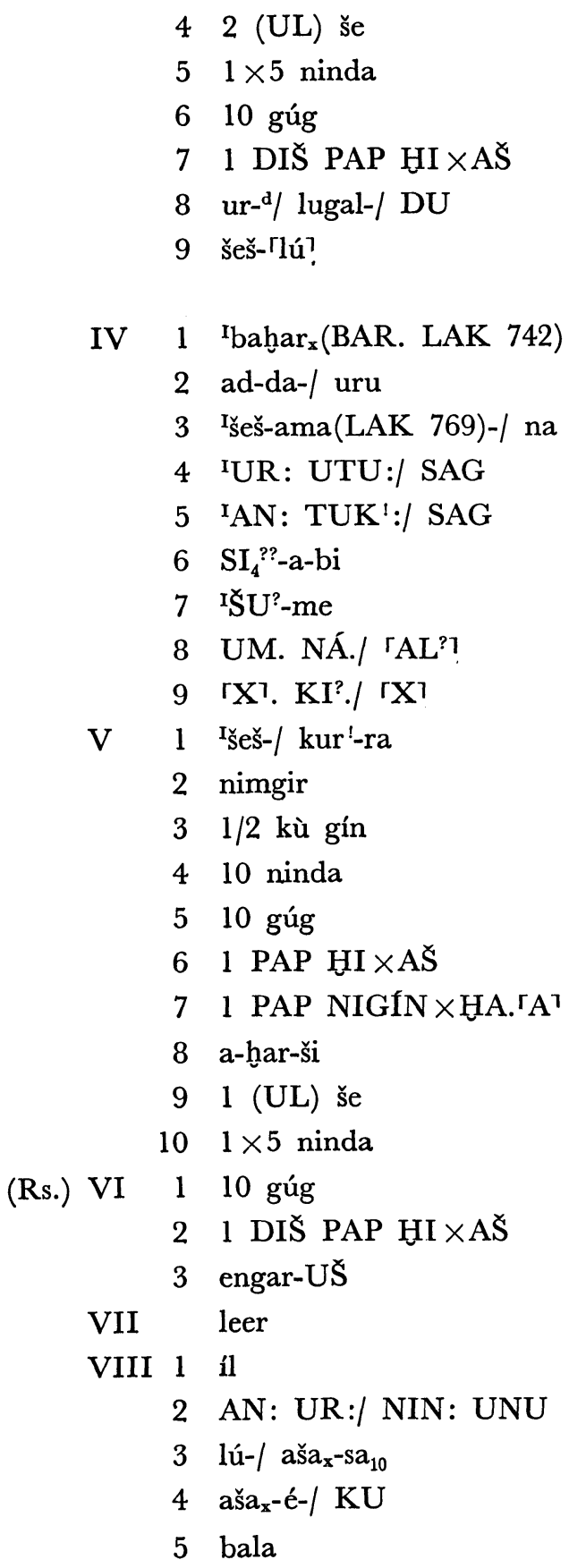

gessen" haben.

2 UL Gerste,

5 Brote,

10 "Gebäcke"

(und) $1+$ DIS PAP H. (sind)

(für) Urlugal-DU,

den "Bruder des Mannes (od. der

Männer)".

IBahar,

Adda'uru,

IŠeš'amana,

'Utu'ursag,

'Sag'antuk,

S.,

IS.,

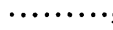

'Šš̌kurra,

der Herold (sind die Zeuge dafür.)

1/2 Seqel Silber,

10 Brote,

10 "Gebäcke,"

1 PAP H. (und)

1 PAP N. (sind)

(für) Aharši.

1 UL Gerste,

5 Brote,

10 "Gebäcke"

(und) $1+$ DIŠ PAP H. (sind)

(für) den Engar-US.

Il

(und) Urnin'unu (sind)

die Käufer des Feldes.

(Flur) Feld des ... -Hauses.

Bala-Amt 
6 INIM:DA:/ AN:SÜD:/ ZI des Inimsudazi.

\section{Kommentar:}

I 2: Zu dieser Lesung aša für das Zeichen GÁN, s. M. Civil, Journal of the Cuneiform Studies 25 (1973), $171 \mathrm{f}$. und M. Powell, ibid., $178 \mathrm{ff}$.

II 4: Es ist ohne Zweifel, daß in unserem Text wie in vielen anderen ähnlichen kein Zahlzeichen vor dem Zeichen TÚG steht. Dies ist durch das Photo des Originals gesichert. Vgl. G. und W. Farber, WO 8, S. 182.

II 6: Für gúg, s. W. Römer, Sumerische 'Königshymnen' der Isin-Zeit, Leiden 1965, S. 184.

II 7: Für PAP als Maßeinheit, s. J. Krecher, $Z A$ 63, $179 \mathrm{f}$.

II 10: Gudu erscheint nirgends in anderen farazeitlichen Kaufverträgen. Vgl. F. Pomponio's Liste in Acta Sumerologica (=ASJ) 5 (1983), $134 \mathrm{ff}$.

III 1: Einige Leute mit dem Namen Nammah sind in Urkunden der Zeit mit verschiedenen Berufnamen belegt; es ist trotzdem schwierig, Nammah in unserem Text $\mathrm{zu}$ identifizieren.

III 2: Diese Person ist hier zum ersten Mal belegt. Gudu, Nammah und Nin'ebalagni sind die gemeinsamen Verkäufer des Feldes, aber leider können wir ihre konkrete, gegenseitige Verwandtschaft nicht wissen. Vgl. J. Krecher, ZA 63, 169 ff.

III 7: Wie man DIŠ versteht, ist noch eine offene Frage; dazu s. vorläufig J. Krecher, ibid., 180.

III 8: Dies ist der erste Beleg dieses Namens, wenn unsere Lesung des letzten Zeichens richtig ist. Das Photo des Originals zeigt ein klares Zeichen DU.

III 9: Zur Interpretation des Terminus šeš-lú, s. zuletzt J. Krecher, ibid., $170 \mathrm{f}$. Betreffs lú in dem Terminus halte ich von den drei Möglichkeiten, die Krecher vorschlug, die erste für wahrscheinlichst, weil die so bezeichnete Person (manchmal auch Pl.) dicht hinter den Verkäufern folgt und gerade vor den Zeugen steht. Dies deutet an, daß lú sich auf die davorstehenden Leute (d. h. Verkäufer) oder mindestens auf einen von ihnen beziehen soll. Sonst wäre die betreffende Person nötig, mit irgendeinem Attribut wie Personennamen, Berufsbezeichnung usw. noch konkreter determiniert zu werden. F. Pomponio, a. a. O., deutet šeš-lú als einen Personennamen. Dagegen wurde nin-lú, die in Texten belegt ist, in die Liste nicht eingtragen. 
IV 1: BAR. EDEN (LAK 742) ist hier keine Berufsbezeichnung, sondern ein Personenname wie in Krecher, $Z A$ 63, 207: 4 III 10; dazu s. Krecher's

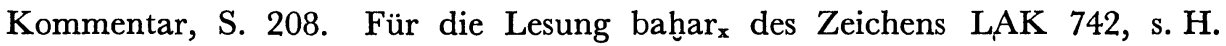
Waetzoldt, WO 6 (1971), $8 \mathrm{f}$.

IV 2: Betreffs des Fehlens des Personenkeil vor einem Namen in den Zeugenlisten s. zuletzt F. Pomponio, a. a. O., $144 \mathrm{f}$ : Anm. 15. Ad-da-uru ist belegt auch in $M V \mathcal{N} 10,84$ III 9-11 wie folgt: ad-da-ki-a, ad-da-uru, é-al-la.

IV 6: Das erste Zeichen ist vielleicht identisch mit LAK 86, d. h. SI $_{4}$. Ein $\mathrm{SI}_{4}$-a-bi als ein Zeuge erscheint in $M V \mathcal{N} 10,86$ IV 5.

IV 8: Unverstehbar.

IV 9: Es fehlt unserem Text an dem Terminus lú-ki-inim "Zeugen", das eigentlich in V 3 zu erwarten ist. Das Photo des Originals zeigt drei unklare Zeichen, die möglicherweise Rasuren von lú-ki-inim sind. Der Schreiber, der merkte, noch einen Mann Seškurra zur Zeugenliste hinzuzufügen, muß vielleicht diese Zeile rasiert aber danach fehlerhaft vergessen haben, in V 3 den Terminus zu schreiben. Auch dem Text SR 9 fehlt er.

V 8: Aharši ist in anderen Urkunden entweder dub-sar (SR 9 IV 2; $M V \mathcal{N}$ 10, 84 VI 1; Krecher, $Z A$ 63, 207: 4 VI 1) oder dub-sar-aša (Krecher, ibid., 196: 1 VIII 7).

VI 3: Zur Funktion eines engar-UŠ s. Krecher, ibid., $175 \mathrm{f}$.

VIII 1 und 2: Daß in unserem Text Il und Urnin'unu die gemeinsamen Käufer des Feldes sind, spricht für die Zugehörigkeit des Textes, Krecher, ibid., 212: $5 \mathrm{zu}$ den Fara-Texten. Vgl. Farber, WO 8, 179, Anm. 2.

VIII 4: KU ist nicht zweifelsfrei. Es mag auch pú/túl "Brunnen" sein, obwohl weder ein Flurname aša $a_{\mathbf{x}}$-é-KU noch aša $\mathbf{x}_{\mathbf{x}}$-é-pú in Urkunden belegt ist.

VIII 5: Zu bala s. Krecher, $Z A$ 63, $181 \mathrm{ff}$. und Farber, $W O$ 8, 183.

Ich hoffe eine möglichst schnelle Publikation der originalen Tontafel, die vielleicht einige ungelöste Probleme klarmachen mag.

\section{Anmerkungen}

(1) Edzard, D. O., Sumerische Rechtsurkunden des III. Jahrtausends aus der Zeit vor der III. Dynastie von Ur, München 1968; J. Krecher, Neue sumerische Rechtsurkunden des 3. Jahrtausends, Zeitschrift für Assyriologie 63 (1973), 145-271.

(2) G. und W. Farber, Ein neuer Feldkaufvertrag aus Fāra, Die Welt des Orients 8 (1975-76), 178-84; J.-P. Grégoire, Materiali per il vocabolario neosumerico, vol. X (1981), Nr. 82-86. Leider habe ich Fara-Text(e) in Orientalia NS 44 nicht zur Verfügung gehabt. 